\title{
MAKING A DOMINATING SET OF A GRAPH CONNECTED
}

\author{
HengZHe Li \\ College of Mathematics and Information Science \\ Henan Normal University, Xinxiang 453007, P.R. China \\ e-mail: lhz@htu.cn \\ BAOYINDURENG WU \\ College of Mathematics and System Sciences \\ Xinjiang University, Urumqi 830046, P.R. China \\ e-mail: baoywu@163.com \\ AND \\ WEIHUA YANG \\ School of Mathematical Science \\ Taiyuan University of Technology \\ Shanxi Taiyuan 030024, P.R. China \\ e-mail: ywh222@163.com
}

\begin{abstract}
Let $G=(V, E)$ be a graph and $S \subseteq V$. We say that $S$ is a dominating set of $G$, if each vertex in $V \backslash S$ has a neighbor in $S$. Moreover, we say that $S$ is a connected (respectively, 2-edge connected or 2-connected) dominating set of $G$ if $G[S]$ is connected (respectively, 2-edge connected or 2-connected). The domination (respectively, connected domination, or 2edge connected domination, or 2-connected domination) number of $G$ is the cardinality of a minimum dominating (respectively, connected dominating, or 2-edge connected dominating, or 2-connected dominating) set of $G$, and is denoted $\gamma(G)$ (respectively $\gamma_{1}(G)$, or $\gamma_{2}^{\prime}(G)$, or $\gamma_{2}(G)$ ). A well-known result of Duchet and Meyniel states that $\gamma_{1}(G) \leq 3 \gamma(G)-2$ for any connected graph $G$. We show that if $\gamma(G) \geq 2$, then $\gamma_{2}^{\prime}(G) \leq 5 \gamma(G)-4$ when $G$ is a 2-edge connected graph and $\gamma_{2}(G) \leq 11 \gamma(G)-13$ when $G$ is a 2-connected triangle-free graph.
\end{abstract}

Keywords: independent set, dominating set, connected dominating set.

2010 Mathematics Subject Classification: 05C69. 


\section{REFERENCES}

[1] L. Arseneau, A. Finbow, B. Hartnell, A. Hynick, D. MacLean and L. O'Sullivan, On minimal connected dominating sets, J. Combin. Math. Combin. Comput. 24 (1997) $185-191$.

[2] C. Bo and B. Liu, Some inequalities about the connected domination number, Discrete Math. 159 (1996) 241-245.

doi:10.1016/0012-365X(95)00088-E

[3] J.A. Bondy and U.S.R. Murty, Graph Theory (GTM 244, Springer, London, 2008).

[4] M. Chellali, O. Favaron, A. Hansberg and L. Volkmann, $k$-domination and $k$-independence in graphs: A survey, Graphs Combin. 28 (2012) 1-55. doi:10.1007/s00373-011-1040-3

[5] C.J. Colbourn and L.K. Stewart, Permutaion graphs: Connected domination and Steiner trees, Discrete Math. 86 (1990) 179-189. doi:10.1016/0012-365X(90)90359-P

[6] D.-Z. Du and P.-J. Wan, Connected Dominating Set: Theory and Applications (Springer, New York, 2013). doi:10.1007/978-1-4614-5242-3

[7] Y.L. Du and H.W. Du, A new bound on maximum independent set and minimum connected dominating set in unit disk graphs, J. Comb. Optim. 30 (2015) 1173-1179. doi:10.1007/s10878-013-9690-0

[8] P. Duchet and H. Meyniel, On Hadwiger's number and the stability number, NorthHolland Math. Studies 62 (1982) 71-73. doi:10.1016/S0304-0208(08)73549-7

[9] S. Guha and S. Khuller, Approximation algorithms for connected dominating sets, Algorithmica 20(1998) 374-387. doi:10.1007/PL00009201

[10] T.W. Haynes, S.T. Hedetniemi and P.J. Slater, Domination in Graphs: The Theory (Marcel Dekker, New York, 1997).

[11] T.W. Haynes, S.T. Hedetniemi and P.J. Slater, Domination in Graphs: Selected Topics (Marcel Dekker, New York, 1997).

[12] M. Li, P.-J. Wan and F. Yao, Tighter approximation bounds for minimum CDS in unit disk graphs, Algorithmica 61 (2011) 1000-1021. doi:10.1007/s00453-011-9512-7

[13] X. Li and Z. Zhang, Two algorithms for minimum 2-connected $r$-hop dominating set, Inform. Process. Lett. 110 (2010) 986-991. doi:10.1016/j.ipl.2010.08.008

[14] M. Moscarini, Doubly chordal graphs, Steiner trees, and connected domination, Networks 23 (1993) 59-69. doi:10.1002/net.3230230108 
[15] E. Sampathkumar and H.B. Walikar, The connected domination number of a graphs, J. Math. Phys. Sci. 13 (1979) 607-613.

[16] E. Vigoda, Lecture Notes on a Parallel Algorithm for Generating a Maximal Independent Set, Georgia Institute of Technology, last updated for 7530 - Randomized Algorithms, Spring 2010.

[17] P.-J. Wan, L. Wang and F. Yao, Two-phased approximation algorithms for minimum CDS in wireless ad hoc networks, in: IEEE ICDCS (2008) 337-344. doi:10.1109/ICDCS.2008.15

[18] K. White, M. Farber and W.R. Pulleyblank, Steiner trees, connected domination and strongly chordal graphs, Networks 15 (1985) 109-124. doi:10.1002/net.3230150109

[19] W. Wu, H. Du, X. Jia, Y. Li and S. Huang, Minimum connected dominating sets and maximal independent sets in unit disk graphs, Theoret. Comput. Sci. 352 (2006) $1-7$.

doi:10.1016/j.tcs.2005.08.037

[20] W. Wu, X. Gao, P.M. Pardalos and D.-Z. Du, Wireless networking, dominating and packing, Optim. Lett. 4 (2010) 347-358.

doi:10.1007/s11590-009-0151-8

Received 20 February 2016

Revised 3 March 2017

Accepted 8 March 2017 